AperTO - Archivio Istituzionale Open Access dell'Università di Torino

\title{
Genericity of the multibump dynamics for almost periodic Duffing-like systems
}

\section{This is a pre print version of the following article:}

Original Citation:

Availability:

This version is available http://hdl.handle.net/2318/110415

since

Published version:

DOI:10.1017/S0308210500030985

Terms of use:

Open Access

Anyone can freely access the full text of works made available as "Open Access". Works made available under a Creative Commons license can be used according to the terms and conditions of said license. Use of all other works requires consent of the right holder (author or publisher) if not exempted from copyright protection by the applicable law. 


\title{
Genericity of the multibump dynamics for almost periodic Duffing-like systems
}

\author{
Francesca Alessio ${ }^{1}$, Paolo Caldiroli ${ }^{2}$ and Piero Montecchiari ${ }^{3}$
}

\author{
${ }^{1}$ Dipartimento di Matematica del Politecnico di Torino \\ Corso Duca degli Abruzzi, 24 - I 10129 Torino, e-mail: alessio@dm.unito.it \\ ${ }^{2}$ Scuola Internazionale Superiore di Studi Avanzati \\ via Beirut, 2-4 - I 34013 Trieste, e-mail: paolocal@sissa.it \\ ${ }^{3}$ Dipartimento di Matematica dell'Università di Trieste \\ Piazzale Europa, 1 - I 34127 Trieste, e-mail: montec@univ.trieste.it
}

\begin{abstract}
In this paper we consider "slowly" oscillating perturbations of almost periodic Duffinglike systems, i.e., systems of the form $\ddot{u}=u-(a(t)+\alpha(\omega t)) W^{\prime}(u), t \in \mathbf{R}, u \in \mathbf{R}^{N}$ where $W \in$ $C^{2 N}\left(\mathbf{R}^{N}, \mathbf{R}\right)$ is superquadratic and $a$ and $\alpha$ are positive and almost periodic. By variational methods, we prove that if $\omega>0$ is small enough then the system admits a multibump dynamics. As a corollary we get that the system $\ddot{u}=u-a(t) W^{\prime}(u), t \in \mathbf{R}, u \in \mathbf{R}^{N}$ admits multibump solutions whenever $a \in \mathcal{A}$, where $\mathcal{A}$ is an open dense subset of $\{a \in C(\mathbf{R}, \mathbf{R}) \mid a$ is almost periodic and $a(t)>$ $0, \forall t \in \mathbf{R}\}$.
\end{abstract}

Key Words. Lagrangian systems, almost periodicity, minimax methods, slow oscillations, multibump dynamics, genericity.

\footnotetext{
${ }^{2}$ Supported by the European Community (contract no. ERBFMBICT961313)

${ }^{3}$ Partially supported by CNR, Consiglio Nazionale delle Ricerche
} 


\section{Introduction}

In this paper we consider the class of Duffing-like systems

$$
\ddot{u}=u-a(t) W^{\prime}(u) \quad t \in \mathbf{R}, u \in \mathbf{R}^{N}
$$

where we assume that $a \in C(\mathbf{R}, \mathbf{R})$ is positive and almost periodic and

$(W 1) W \in C^{2 N}\left(\mathbf{R}^{N}, \mathbf{R}\right)$,

$(W 2)$ there exists $\theta>2$ such that $0<\theta W(x) \leq W^{\prime}(x) x$ for any $x \neq 0$,

$(W 3) W^{\prime}(x) x<W^{\prime \prime}(x) x x$ for any $x \neq 0$.

We recall that a function $a \in C(\mathbf{R}, \mathbf{R})$ is almost periodic if for any $\varepsilon>0$ there exists $L_{\varepsilon}>0$ such that any interval $I \subset \mathbf{R}$ of lenght $L_{\varepsilon}$ contains an $\varepsilon$-period of $a$, that is, a $\tau \in \mathbf{R}$ for which $\sup _{t \in \mathbf{R}}|a(t-\tau)-a(t)| \leq \varepsilon$. We will denote by $P_{\varepsilon}(a)$ the set of $\varepsilon$-periods of $a$.

Note that the Duffing system, i.e., the system $\left(D_{a}\right)$ with $W(x)=\frac{|x|^{4}}{4}$, satisfies the above assumptions. By $(W 2)$, it follows in particular that $W^{\prime}(0)=$ 0 and $W^{\prime \prime}(0)=0$ and therefore that the origin in the phase space is a hyperbolic rest point for $\left(D_{a}\right)$. A non zero solution $u \in C^{2}\left(\mathbf{R}, \mathbf{R}^{N}\right)$ of $\left(D_{a}\right)$ is called homoclinic to the origin if $u(t) \rightarrow 0$ and $\dot{u}(t) \rightarrow 0$ as $t \rightarrow \pm \infty$.

Starting with [Bol], [BG], [CZES] and [R1], the existence and multiplicity of homoclinic solutions for Hamiltonian systems has been studied by variational methods. The variational approach has permitted to study systems with different time dependence of the Hamiltonian. We mention [CZES], [R1], [HW], [S1], [CZR], [S2], [T], [Be1], [CM], [Be2], [Be3], [CS], [R3] and [MNT2] for the periodic case, [BB], [STT], [CZMN], [R2], [Sp] and [MNT1] for the almost periodic and recurrent cases.

In [S2] it was introduced a novel minimax method which has permitted to prove shadowing-like Lemmas and consequently to show the existence of a class of solutions, called multibump solutions, whose presence displays some chaotic features of the dynamics. Precisely the presence of an approximate Bernoulli shift and the positive topological entropy. The use of this method has then permitted on one hand to consider non degeneracy conditions on the set of "generating" homoclinic solutions, weaker than the classical transversality intersection between the stable and unstable manifolds, see [Be1], [BS], [CS], [R3] and [MNT2] (for the classical geometrical 
approach of the Dynamical System Theory see e.g. [W1]). On the other hand, as we said above, the variational methods allow to extend these results to more generally time dependent Hamiltonian systems, see [CZMN], [R2], [MNT1] and [AM]. In these cases the geometrical approach is hardly applicable since the dynamics is not well described by a suitable Poincaré map (like the time-T map in the T-periodic case) and it is not possible to apply directly the Smale Birkhoff Theorem. However we quote [MS] for a result in this direction for almost periodic perturbations of autonomous second order equations. We also mention [Sc] where a shadowing Lemma for almost periodic systems is proved using the notion of exponential dichotomy, firstly introduced in $[\mathrm{P}]$.

We point out that all the above results are based on some a priori discreteness assumptions on the set of homoclinic solutions, that are in general difficult to test. The use of the Melnikov function, see $[\mathrm{M}]$, permits to check them for periodic perturbations of planar autonomous systems. In this direction we mention $[\mathrm{AB}]$ and $[\mathrm{BBo}]$, which generalize the Melnikov method to systems of any dimension and with arbitrary time dependence of the perturbative term. A refined use of the Melnikov method permits to detect chaotic properties for perturbated systems when the periodic perturbative term is not necessarily small in $L^{\infty}$ norm but oscillates fastly (see [An]) or slowly (see [W1], [W2], [WH] and the references therein). All these results are based on the fact that the unperturbed system is an integrable Hamiltonian system having a homoclinic solution which is asked to be non degenerate modulo time translations.

In the slowly oscillating case it is possible to bypass the use of Melnikovtype techniques, checking the discreteness conditions on the set of the homoclinic solutions with a global variational approach. This is done without making assumptions on the set of homoclinics of the unperturbed system by exploiting concentration phenomena of the solutions with respect to the slow oscillations of the Hamiltonian, as in $[\mathrm{AM}]$. Results in this direction for the Schrödinger equation are contained for instance in $[\mathrm{ABC}],[\mathrm{DPF}],[\mathrm{G}]$ and $[\mathrm{L}]$, where analogous concentration phenomena occur.

Considering the system $\ddot{u}=u-\alpha(\omega t) W^{\prime}(u)$, with $\alpha \in C(\mathbf{R}, \mathbf{R})$ non constant, positive and almost periodic, we will see that the argument in $[\mathrm{AM}]$ can be adapted to prove that if the system is slowly oscillating, i.e., if $\omega>0$ is sufficiently small, then it admits a multibump dynamics.

In fact, in this paper we consider the (non integrable) system $\left(D_{a}\right)$ on which we have no a priori knowledge of its global dynamics and we show 
that if we perturb it with a slowly oscillating term then the new system exhibits a multibump dynamics. More precisely, considered the system

$$
\ddot{u}=u-(a(t)+\alpha(\omega t)) W^{\prime}(u), \quad t \in \mathbf{R}, u \in \mathbf{R}^{N}
$$

we prove

Theorem 1.1 For all $a \in C(\mathbf{R}, \mathbf{R})$ non negative, almost periodic and for all $\alpha \in C(\mathbf{R}, \mathbf{R})$ non costant, positive, almost periodic there exists $\bar{\omega}>0$ such that for every $\omega \in(0, \bar{\omega})$ the system $\left(D_{\omega}\right)$ admits multibump solutions. Precisely, there exists a compact set $K_{\omega} \subset C^{2}\left(\mathbf{R}, \mathbf{R}^{N}\right)$ of homoclinic solutions of $\left(D_{\omega}\right)$ for which for any $r>0$ there is $N_{r}>0$ and $\varepsilon_{r}>0$ such that for any sequence $\left(p_{j}\right) \subset P_{\varepsilon_{r}}(a(\cdot)+\alpha(\omega \cdot))$, with $p_{j+1}-p_{j} \geq N_{r}$, and $\sigma=\left(\sigma_{j}\right) \subset\{0,1\}^{\mathbf{Z}}$, there exists a solution $v_{\sigma}$ of $\left(D_{\omega}\right)$ verifying

$$
\inf _{u \in K_{\omega}}\left\|v_{\sigma}-\sigma_{j} u\left(\cdot-p_{j}\right)\right\|_{C^{1}\left(I_{j}\right)}<r
$$

for any $j \in \mathbf{Z}$, where $I_{j}=\left[\frac{p_{j-1}+p_{j}}{2}, \frac{p_{j}+p_{j+1}}{2}\right]$. In addition $v_{\sigma}$ is a homoclinic solution whenever $\sigma_{j}=0$ definitively.

By Theorem 1.1, since no condition on the $L^{\infty}$ norm of $\alpha$ is required, we plainly obtain that the set of almost periodic functions $a$ for which the system $\left(D_{a}\right)$ admits multibump dynamics is dense with respect to the $L^{\infty}$ topology in the set of positive almost periodic continuous functions.

In fact, the result stated in Theorem 1.1 is obtained by proving a discretness condition on the set of homoclinic solutions of $\left(D_{\omega}\right)$. This condition turns out to be stable with respect to perturbations of the field (see $[\mathrm{MN}]$, $[\mathrm{Be} 2]$ and $[\mathrm{Be} 3]$ for analogous result in the periodic case). In particular, if this assumption is satisfied for the system $\left(D_{a}\right)$, then it is also verified for the system $\ddot{u}=u-(a(t)+h(t)) W^{\prime}(u)$, provided that $\|h\|_{L^{\infty}}$ is sufficiently small. Then we obtain

Theorem 1.2 There exists an open dense subset $\mathcal{A}$ of $\{a \in C(\mathbf{R}, \mathbf{R}) \mid a$ is almost periodic and $a(t)>0, \forall t \in \mathbf{R}\}$ such that for any $a \in \mathcal{A}$ the system $\left(D_{a}\right)$ admits multibump solutions.

This result expresses the genericity of a chaotic behaviour for the continuous flows associated to the class of systems $\left(D_{a}\right)$. We refer to [PT] and [W1] for other genericity results in the framework of the geometric theory of chaotic dynamical systems, as for instance the classical generic property 
of transversal intersection between the stable and unstable manifolds for diffeomorphisms on manifolds, stated by the Kupka-Smale Theorem (see $[\mathrm{W} 1])$.

Acknowledgement. This work was done while the authors were visiting CEREMADE. They wish to thank CEREMADE for the kind hospitality.

\section{Preliminary results}

In this section we state some properties shared by a class of functionals defined on the Sobolev space $X=H^{1}\left(\mathbf{R}, \mathbf{R}^{N}\right)$ of the type

$$
\varphi(u)=\frac{1}{2}\|u\|^{2}-\int_{\mathbf{R}} k(t) W(u(t)) d t
$$

where $\|u\|=\left(\int_{\mathbf{R}}|\dot{u}|^{2}+|u|^{2}\right)^{\frac{1}{2}}$ is the standard norm of $X, W$ satisfies $(W 1)$, $(W 2)$ and (W3) and $k \in C(\mathbf{R}, \mathbf{R})$ is positive and bounded.

It is standard to check that, by $(W 1)$ and $(W 2), \varphi \in C^{2 N}(X, \mathbf{R})$ and that critical points of $\varphi$ are exactly (classical) solutions of the system $-\ddot{u}+u=$ $k(t) W^{\prime}(u)$ satisfying $u(t) \rightarrow 0$ and $\dot{u}(t) \rightarrow 0$ as $t \rightarrow \pm \infty$.

Even if we are mainly interested in the case $k(t)=a(t)+\alpha(\omega t)$, with $a, \alpha \in C(\mathbf{R}, \mathbf{R})$ fixed as in the statement of Theorem 1.1 and $\omega>0$ small enough, actually we have to handle a family of functionals corresponding to the "problems at infinity", in which $k(t)=b(t)+\alpha(\omega t)$ or $k(t)=b(t)+\beta$, where $\beta \in[\inf \alpha, \sup \alpha]$ and $b \in H(a)=\overline{\{a(\cdot-\tau): \tau \in \mathbf{R}\}} \|^{\|\|_{L^{\infty}}}$.

Therefore, in this section, we consider the class $\mathcal{F}$ of functionals like (2.1) with $k \in C(\mathbf{R}, \mathbf{R})$ satisfying $0<k(t) \leq \bar{a}$, where $\bar{a}=\|a\|_{L^{\infty}}+\|\alpha\|_{L^{\infty}}$, and we prove some preliminary properties of the functionals $\varphi \in \mathcal{F}$ which are uniform with respect to the class $\mathcal{F}$.

First, we recall a result concerning the behavior of any functional $\varphi \in \mathcal{F}$ at 0 .

Lemma 2.1 There exists $\bar{\rho}>0$ such that for any interval $I \subseteq \mathbf{R}$ with length $|I| \geq 1$ and for any $\varphi \in \mathcal{F}$, if $\|u\|_{I} \leq 2 \bar{\rho}$, then $\varphi_{I}(u) \geq \frac{1}{4}\|u\|_{I}^{2}$ and $\varphi_{I}^{\prime}(u) u \geq$ $\frac{3}{4}\|u\|_{I}^{2}$, where $\|u\|_{I}=\left(\int_{I}|\dot{u}|^{2}+|u|^{2}\right)^{\frac{1}{2}}$ and $\varphi_{I}(u)=\frac{1}{2}\|u\|_{I}^{2}-\int_{I} k(t) W(u(t)) d t$.

As an immediate consequence we get: 
Lemma 2.2 For any $\varphi \in \mathcal{F}$ we have:

(i) 0 is a strict local minimum point for $\varphi$,

(ii) if $\left(u_{n}\right) \subset X$ is such that $\lim \sup \left\|u_{n}\right\| \leq 2 \bar{\rho}$ and $\varphi^{\prime}\left(u_{n}\right) \rightarrow 0$ then $u_{n} \rightarrow 0$,

(iii) $\inf \left\{\|u\|: u \in \mathcal{K}_{\varphi}\right\} \geq 2 \bar{\rho}$,

where $\mathcal{K}_{\varphi}=\left\{u \in X: \varphi^{\prime}(u)=0, u \neq 0\right\}$ (we agree that inf $\emptyset=+\infty$ ).

Remark 2.1 The assumption (W2) implies that for every $u \in X$

$$
\left(\frac{1}{2}-\frac{1}{\theta}\right)\|u\|^{2} \leq \varphi(u)+\frac{1}{\theta}\left\|\varphi^{\prime}(u)\right\|\|u\| .
$$

Then, by Lemma 2.2 (iii), we get $\inf _{\mathcal{K}_{\varphi}} \varphi \geq\left(2-\frac{4}{\theta}\right) \bar{\rho}^{2}=\bar{\lambda}>0$ for any $\varphi \in \mathcal{F}$.

By $(W 2), W(x) \geq W\left(\frac{x}{|x|}\right)|x|^{\theta}$ for $|x| \geq 1$. Then, there exists $u_{1} \in X$ such that $\varphi\left(u_{1}\right)<0$ for every $\varphi \in \mathcal{F}$. Hence, by Lemma $2.2(i)$, any functional $\varphi \in \mathcal{F}$ has a mountain pass geometry and, setting

$$
c_{\varphi}=\inf _{\gamma \in \Gamma} \sup _{s \in[0,1]} \varphi(\gamma(s))
$$

where $\Gamma=\left\{\gamma \in C([0,1], X): \gamma(0)=0, \gamma(1)=u_{1}\right\}$, we have that $c_{\varphi} \geq$ $\frac{1}{4} \bar{\rho}^{2}>0$ for every $\varphi \in \mathcal{F}$ and, by the mountain pass Lemma, there exists a sequence $\left(u_{n}\right) \subset X$ such that $\varphi\left(u_{n}\right) \rightarrow c_{\varphi}$ and $\varphi^{\prime}\left(u_{n}\right) \rightarrow 0$.

Remark 2.2 By $(W 3)$ for every $u \in X \backslash\{0\}$ there exists a unique $s(u)>0$ such that $\left.\frac{d}{d s} \varphi(s u)\right|_{s=s(u)}=0$ and hence $\varphi(s(u) u)=\max _{s \geq 0} \varphi(s u)$. In this case we have $c_{\varphi}=\inf _{u \in X \backslash\{0\}} \sup _{s \geq 0} \varphi(s u)$ and $\inf _{\mathcal{K}_{\varphi}} \varphi \geq c_{\varphi}$.

Considered a functional $\varphi \in \mathcal{F}$, we are interested in studying Palais Smale sequences (briefly $P S$ sequences) for $\varphi$, i.e., sequences $\left(u_{n}\right) \subset X$ such that $\left(\varphi\left(u_{n}\right)\right)$ is bounded and $\left\|\varphi^{\prime}\left(u_{n}\right)\right\| \rightarrow 0$.

Remark 2.3 By (2.2), any $P S$ sequence $\left(u_{n}\right)$ for $\varphi$ is bounded. Moreover, $\liminf \varphi\left(u_{n}\right) \geq 0$ and if $\lim \sup \varphi\left(u_{n}\right)<\bar{\lambda}$ then $u_{n} \rightarrow 0$.

We recall a first result well known in the literature (see e.g. [CZR]).

Lemma 2.3 If $\left(u_{n}\right) \subset X$ is a PS sequence for $\varphi$ weakly converging to $u \in X$, then: 
(i) $\varphi(u) \leq \liminf \varphi\left(u_{n}\right)$ and $\varphi^{\prime}(u)=0$,

(ii) $\left(u_{n}-u\right)$ is a PS sequence for $\varphi$ with $\lim \sup \varphi\left(u_{n}-u\right) \leq \lim \sup \varphi\left(u_{n}\right)-$ $\varphi(u)$,

(iii) $u_{n} \rightarrow u$ strongly in $H_{l o c}^{1}\left(\mathbf{R}, \mathbf{R}^{N}\right)$.

By Lemmas 2.1 and 2.2, it can be proved that we lose compactness of those $P S$ sequences $\left(u_{n}\right)$ which "carry mass" at infinity, i.e., there exists a sequence $\left(t_{n}\right) \subset \mathbf{R}$ such that $\left|t_{n}\right| \rightarrow \infty$ and liminf $\left\|u_{n}\right\|_{|t| \geq t_{n}} \geq 2 \bar{\rho}$. In order to obtain compactness results it is therefore useful to introduce the function $T: X \rightarrow \mathbf{R} \cup\{-\infty\}$ defined in the following way:

$$
T(u)= \begin{cases}\sup \left\{T \in \mathbf{R}:\|u\|_{t>T}=\bar{\rho}\right\} & \text { if }\|u\|>\bar{\rho} \\ -\infty & \text { otherwise } .\end{cases}
$$

This function is well defined since the mapping $T \mapsto\|u\|_{t>T}$ is non increasing, $\|u\|_{t>T} \rightarrow 0$ as $T \rightarrow+\infty$ and $\|u\|_{t>T} \rightarrow\|u\|$ as $T \rightarrow-\infty$. Moreover, if $\|u\|>\bar{\rho}$ then $T(u) \in \mathbf{R}$ and $\|u\|_{t>T(u)}=\bar{\rho}$. We also note that $T(u(\cdot-s))=T(u)+s$ for every $u \in X$ and $s \in \mathbf{R}$.

The function $T$ is not continuous on $X$ but satisfies

Lemma 2.4 If $u_{n} \rightarrow u$ weakly in $X$ and $u(T(u)) \neq 0$, then $\liminf T\left(u_{n}\right) \geq$ $T(u)$. Moreover, if in addition $u_{n} \rightarrow u$ strongly in $H^{1}\left((T(u),+\infty), \mathbf{R}^{N}\right)$ then $T\left(u_{n}\right) \rightarrow T(u)$.

Proof. By contradiction, suppose that $T(u)>\lim \inf T\left(u_{n}\right)=\underline{T} \geq-\infty$. Then, for any $T \in(\underline{T}, T(u))$ there exists $n_{T} \in \mathbf{N}$ such that $T\left(u_{n}\right) \leq T$ for every $n \geq n_{T}$. Hence

$$
\begin{aligned}
\bar{\rho}^{2} & =\|u\|_{t>T(u)}^{2}=\|u\|_{t>T}^{2}-\|u\|_{[T, T(u)]}^{2} \leq \liminf \left\|u_{n}\right\|_{t>T}^{2}-\|u\|_{[T, T(u)]}^{2} \\
& \leq \liminf \left\|u_{n}\right\|_{t>T\left(u_{n}\right)}^{2}-\|u\|_{[T, T(u)]}^{2}=\bar{\rho}^{2}-\|u\|_{[T, T(u)]}^{2}
\end{aligned}
$$

that, since $X \subset C\left(\mathbf{R}, \mathbf{R}^{N}\right)$, implies $u(T(u))=0$, contrary to the assumption. To prove the second part we argue in a similar way. If it were $T(u)<$ $\lim \sup T\left(u_{n}\right)=\bar{T}$, then, given any $T \in(T(u), \bar{T})$, there exists $n_{T} \in \mathbf{N}$ such that $T\left(u_{n}\right) \geq T$ for every $n \geq n_{T}$ and thus, since $\left\|u_{n}-u\right\|_{t>T} \rightarrow 0$ and $|u(t)|>0$ in a neighborhood of $T(u)$, we get

$$
\bar{\rho}=\|u\|_{t>T(u)}>\|u\|_{t>T}=\lim \left\|u_{n}\right\|_{t>T} \geq \lim \left\|u_{n}\right\|_{t>T\left(u_{n}\right)}=\bar{\rho},
$$

a contradiction.

We have the following auxiliary result 
Lemma 2.5 For any $b>0$ there exist $\delta>0$ and $\tau>0$ such that for every $u \in\{\varphi \leq b\} \cap\left\{\left\|\varphi^{\prime}\right\| \leq \frac{1}{2} \bar{\rho}\right\}$ with $T(u) \in \mathbf{R}$ it holds that $|u(t)| \geq \delta$ for any $t \in[T(u)-\tau, T(u)]$.

Proof. If not, there exist $b>0$, a sequence $\left(u_{n}\right) \subset\{\varphi \leq b\} \cap\left\{\left\|\varphi^{\prime}\right\| \leq \frac{1}{2} \bar{\rho}\right\}$ and a sequence $\left(t_{n}\right) \subset \mathbf{R}$ such that $t_{n} \leq T\left(u_{n}\right)$ for any $n \in \mathbf{N}, T\left(u_{n}\right)-t_{n} \rightarrow 0$ and $u_{n}\left(t_{n}\right) \rightarrow 0$. We introduce a sequence $\left(v_{n}\right) \subset X$ defined as follows:

$$
v_{n}(t)= \begin{cases}0 & \text { for } t \leq t_{n}-1 \\ \left(t-t_{n}+1\right) u_{n}\left(t_{n}\right) & \text { for } t_{n}-1<t \leq t_{n} \\ u_{n}(t) & \text { for } t>t_{n}\end{cases}
$$

We have $\left\|v_{n}\right\|_{\left[t_{n}-1, t_{n}\right]}=O\left(\left|u_{n}\left(t_{n}\right)\right|\right)$ and, since $\left(u_{n}\right)$ is bounded in $L^{\infty}$, $\int_{t_{n}}^{T\left(u_{n}\right)} k(t) W^{\prime}\left(u_{n}(t)\right) v_{n}(t) d t=O\left(T\left(u_{n}\right)-t_{n}\right)$. Then, since $\left\|u_{n}\right\|_{t>T\left(u_{n}\right)}<2 \bar{\rho}$, we obtain

$\varphi^{\prime}\left(u_{n}\right) v_{n}=\left\|u_{n}\right\|_{t>t_{n}}^{2}-\int_{T\left(u_{n}\right)}^{\infty} k(t) W^{\prime}\left(u_{n}(t)\right) u_{n}(t) d t+o(1) \geq \frac{3}{4}\left\|u_{n}\right\|_{t>t_{n}}^{2}+o(1)$ and $\left\|v_{n}\right\|^{2}=\left\|u_{n}\right\|_{t>t_{n}}^{2}+o(1)$ as $n \rightarrow+\infty$ which leads to the contradiction

$$
\frac{1}{2} \bar{\rho} \geq \varphi^{\prime}\left(u_{n}\right) \frac{v_{n}}{\left\|v_{n}\right\|} \geq \frac{3}{4}\left\|u_{n}\right\|_{t>t_{n}}+o(1) \geq \frac{3}{4} \bar{\rho}+o(1) .
$$

Remark 2.4 From the above proof, one can see that the result stated in Lemma 2.5 holds true uniformly with respect to the class of functionals $\mathcal{F}$. That is, the values $\delta$ and $\tau$ depend only on $b$ but not on the choice of $\varphi \in \mathcal{F}$.

Then we obtain

Lemma 2.6 If $\left(u_{n}\right) \subset X$ is a PS sequence for $\varphi$ weakly converging to $u \in X$ and $\left(T\left(u_{n}\right)\right)$ is bounded, then $u \neq 0$ and $T\left(u_{n}\right) \rightarrow T(u)$.

Proof. Let $b>0$ be such that $\varphi\left(u_{n}\right) \leq b$ for every $n \in \mathbf{N}$. Since for $n \in \mathbf{N}$ large enough, $\left\|\varphi^{\prime}\left(u_{n}\right)\right\| \leq \frac{1}{2} \bar{\rho}$, by Lemma 2.5, there exists $\delta>0$ such that $\left|u_{n}\left(T\left(u_{n}\right)\right)\right| \geq \delta$ for any $n \in \mathbf{N}$ sufficiently large. Since the sequence $\left(T\left(u_{n}\right)\right)$ is bounded, up to a subsequence, it converges to some $T \in \mathbf{R}$ and, by the $L_{l o c}^{\infty}$ convergence, $u_{n}\left(T\left(u_{n}\right)\right) \rightarrow u(T)$. Then $|u(T)| \geq \delta$ and so $u \neq 0$. By Lemma $2.3(i), \varphi^{\prime}(u)=0$. Hence $u \in \mathcal{K}_{\varphi}$ and Lemma $2.2($ iii) implies that $\|u\| \geq 2 \bar{\rho}$. Thus $T(u) \in \mathbf{R}$ and, using again Lemma $2.5, u(T(u)) \neq 0$. To complete the 
proof, it is enough to check that $u_{n} \rightarrow u$ strongly in $H^{1}\left((T(u),+\infty), \mathbf{R}^{N}\right)$ and apply Lemma 2.4. To this aim, let $T \geq \sup T\left(u_{n}\right)$ such that $\|u\|_{t>T}<\frac{\bar{\rho}}{4}$ and consider the cut-off function $\chi_{T}: \mathbf{R} \rightarrow[0,1]$ defined as:

$$
\chi_{T}(t)= \begin{cases}0 & \text { for } t \leq T \\ t-T & \text { for } T<t \leq T+1 \\ 1 & \text { for } t>T+1\end{cases}
$$

Since by Lemma 2.3 (iii) we have $u_{n}-u \rightarrow 0$ in $H_{l o c}^{1}\left(\mathbf{R}, \mathbf{R}^{N}\right)$, it is easy to check $\left\|\varphi^{\prime}\left(u_{n}-u\right)-\varphi^{\prime}\left(\chi_{T}\left(u_{n}-u\right)\right)\right\| \rightarrow 0$. Then, by Lemma $2.3(i i)$, we infer that $\left(\chi_{T}\left(u_{n}-u\right)\right)$ is a $P S$ sequence for $\varphi$. Moreover, since $T \geq \sup T\left(u_{n}\right)$ and $\|u\|_{t>T}<\frac{\bar{\rho}}{4}$, we get

$$
\left\|\chi_{T}\left(u_{n}-u\right)\right\| \leq \sqrt{2}\left\|u_{n}-u\right\|_{t>T} \leq \sqrt{2}\left(\left\|u_{n}\right\|_{t>T}+\|u\|_{t>T}\right) \leq 2 \bar{\rho} .
$$

By Lemma $2.2(i i)$ this implies $\chi_{T}\left(u_{n}-u\right) \rightarrow 0$ and since $u_{n}-u \rightarrow 0$ in $H_{l o c}^{1}\left(\mathbf{R}, \mathbf{R}^{N}\right)$ we conclude that $u_{n}-u \rightarrow 0$ in $H^{1}\left((T(u),+\infty), \mathbf{R}^{N}\right)$.

Then we obtain

Corollary 2.1 Any PS sequence $\left(u_{n}\right)$ for $\varphi \in \mathcal{F}$ with $\lim \sup \varphi\left(u_{n}\right)<c_{\varphi}+\bar{\lambda}$ and $\left(T\left(u_{n}\right)\right)$ bounded is precompact in $X$.

Proof. By Remark 2.3, the sequence $\left(u_{n}\right)$ is bounded in $X$ and then it admits a subsequence, always denoted by $\left(u_{n}\right)$, weakly convergent to some $u \in X$. By Lemmas 2.3 and 2.6, $u \in \mathcal{K}_{\varphi}$ and $\left(u_{n}-u\right)$ is a $P S$ sequence for $\varphi$ with $\lim \sup \varphi\left(u_{n}-u\right)<c_{\varphi}+\bar{\lambda}-\varphi(u)$. By Remark 2.2, $\varphi(u) \geq c_{\varphi}$ and then $\lim \sup \varphi\left(u_{n}-u\right)<\bar{\lambda}$. Then, by Remark $2.3, u_{n} \rightarrow u$.

Moreover we have the folowing locally Lipschitz continuity of $T$ on $\left\{\left\|\varphi^{\prime}\right\| \leq\right.$ $\left.\frac{\bar{\rho}}{2}\right\} \backslash \overline{B_{\bar{\rho}}(0)}$

Lemma 2.7 For any $b>0$ there exist $r>0$ and $L>0$ such that $\mid T\left(u_{1}\right)-$ $T\left(u_{2}\right) \mid \leq L\left\|u_{1}-u_{2}\right\|$ for every $u_{1}, u_{2} \in\{\varphi \leq b\} \cap\left\{\left\|\varphi^{\prime}\right\| \leq \frac{1}{2} \bar{\rho}\right\} \backslash B_{\bar{\rho}}(0)$ with $\left\|u_{1}-u_{2}\right\| \leq r$.

Proof. Let $b>0$ and let $\delta>0$ and $\tau>0$ be given by Lemma 2.5. We set $r=\sqrt{\bar{\rho}^{2}+\delta^{2} \tau}-\bar{\rho}$. Taken $u_{1}, u_{2} \in\left\{\left\|\varphi^{\prime}\right\| \leq \frac{1}{2} \bar{\rho}\right\} \cap\{\varphi \leq b\}$ with $-\infty<$ $T\left(u_{1}\right)<T\left(u_{2}\right)<+\infty$ and $\left\|u_{1}-u_{2}\right\| \leq r$ we have

$$
\bar{\rho}=\left\|u_{1}\right\|_{t>T_{1}} \geq\left\|u_{2}\right\|_{t>T_{1}}-\left\|u_{1}-u_{2}\right\|_{t>T_{1}} \geq \sqrt{\bar{\rho}^{2}+\left\|u_{2}\right\|_{\left[T_{1}, T_{2}\right]}^{2}}-\left\|u_{1}-u_{2}\right\|
$$

and then

$$
\left\|u_{2}\right\|_{\left[T_{1}, T_{2}\right]}^{2} \leq 2 \bar{\rho}\left\|u_{1}-u_{2}\right\|+\left\|u_{1}-u_{2}\right\|^{2}
$$


where $T_{i}=T\left(u_{i}\right)$ for $i=1,2$. If $T_{2}-T_{1}>\tau$, then, by Lemma 2.5, $\left\|u_{2}\right\|_{\left[T_{1}, T_{2}\right]}^{2}>\delta^{2} \tau$ and so, by $(2.4), \delta^{2} \tau<2 \bar{\rho} r+r^{2}$, contrary to the definition of $r$. Hence $T_{2}-T_{1} \leq \tau$ and, by (2.4),

$$
\delta^{2}\left(T_{2}-T_{1}\right) \leq\left\|u_{2}\right\|_{\left[T_{1}, T_{2}\right]}^{2} \leq(2 \bar{\rho}+r)\left\|u_{2}-u_{1}\right\|
$$

and the thesis is proved with $L=\delta^{-2}\left(\bar{\rho}+\sqrt{\bar{\rho}^{2}+\delta^{2} \tau}\right)$.

\section{The perturbated system}

In this section we will study the system $\left(D_{\omega}\right)$ and we prove that if $\omega>0$ is sufficiently small then the set of homoclinic solutions of $\left(D_{\omega}\right)$ satisfies suitable discreteness properties. In fact, considered the action functional

$$
\varphi_{\omega}(u)=\frac{1}{2}\|u\|^{2}-\int_{\mathbf{R}}(a(t)+\alpha(\omega t)) W(u) d t,
$$

assuming that there exist $\bar{r}>0$ and $\tilde{r} \in(0, \bar{r})$ such that $\bar{\alpha}=\alpha(0) \geq$ $\max _{|t| \leq \bar{r}} \alpha(t)>\max _{\tilde{r} \leq|t| \leq \bar{r}} \alpha(t)=\underline{\alpha}$ (not restrictive since $a$ is almost periodic and not constant), we will prove

Theorem 3.1 There exists $\tilde{\omega}>0$ and $\tilde{c}>\sup _{\omega \in(0, \tilde{\omega})} c_{\omega}$ such that

$$
T\left(\mathcal{K}_{\omega} \cap\left\{\varphi_{\omega} \leq \tilde{c}\right\}\right) \cap\left\{t \in \mathbf{R}\left|\frac{\tilde{r}}{\omega} \leq\right| t \mid \leq \frac{\bar{r}}{\omega}\right\}=\emptyset
$$

for all $\omega \in(0, \tilde{\omega})$, where $\mathcal{K}_{\omega}$ is the set of critical points of $\varphi_{\omega}$ and $c_{\omega}$ is the mountain pass level of $\varphi_{\omega}$.

In the next section we will show that if the condition $\left(H_{\omega}\right)$ is satisfied then the system $\left(D_{\omega}\right)$ admits a multibump dynamics.

First we fix some notation. For any $b \in H(a)$ and $\beta \in[\inf \alpha$, sup $\alpha]$, let $\psi_{b \beta}$ be the functional defined as

$$
\psi_{b \beta}(u)=\frac{1}{2}\|u\|^{2}-\int_{\mathbf{R}}(b(t)+\beta) W(u) d t, \quad \forall u \in X .
$$

Note that all the functionals $\varphi_{\omega}$ and $\psi_{b \beta}$ belong to the class $\mathcal{F}$ defined in the previous section. 
Remark 3.1 The almost periodicity of $a$ and Remark 2.2, imply that the mountain pass level $c_{b \beta}$ of the functional $\psi_{b \beta}$ is independent of $b \in H(a)$ (we will denote it by $c_{\beta}$ ) and that for all $\beta$ there exist $b \in H(a)$ and $u \in X$ such that $\psi_{b \beta}^{\prime}(u)=0$ and $\psi_{b \beta}(u)=c_{\beta}$, see e.g. [CZMN].

Moreover, if $\beta_{1}<\beta_{2}$ we have $c_{\beta_{1}}>c_{\beta_{2}}$. Indeed, by Remark 2.2, there exist $b \in H(a)$ and $\gamma \in \Gamma$ such that $\max _{s \in[0,1]} \psi_{b \beta_{1}}(\gamma(s))=c_{\beta_{1}}$. Let $u \in$ range $\gamma$ be such that $\psi_{b \beta_{2}}(u)=\max _{s \in[0,1]} \psi_{b \beta_{2}}(\gamma(s)) \geq c_{\beta_{2}}$. Then $u \not \equiv 0$ and therefore, since $\beta_{1}<\beta_{2}$,

$$
c_{\beta_{2}} \leq \psi_{b \beta_{2}}(u)<\psi_{b \beta_{1}}(u) \leq \max _{s \in[0,1]} \psi_{b \beta_{1}}(\gamma(s))=c_{\beta_{1}} .
$$

We are interested in studying sequences $\left(u_{n}\right) \subset X$ such that $\varphi_{\omega_{n}}^{\prime}\left(u_{n}\right) \rightarrow 0$ and $\left(\varphi_{\omega_{n}}\left(u_{n}\right)\right)$ is bounded for some sequence $\left(\omega_{n}\right) \subset(0,+\infty)$.

First note that by (2.2) these sequences are bounded and therefore weakly precompact in $X$. Moreover, by Lemma 2.1 and Remark 2.3, if $\bar{\rho}>0$ is fixed according to Lemma 2.1, we have

Lemma 3.1 Let $\left(\omega_{n}\right) \subset(0,+\infty)$ and $\left(u_{n}\right) \subset X$ be such that $\varphi_{\omega_{n}}^{\prime}\left(u_{n}\right) \rightarrow 0$. Then we have

(i) $\lim \inf \varphi_{\omega_{n}}\left(u_{n}\right) \geq 0$ and

(ii) if $\lim \sup \left\|u_{n}\right\| \leq 2 \bar{\rho}$ or $\lim \sup \varphi_{\omega_{n}}\left(u_{n}\right)<\bar{\lambda}$ then $u_{n} \rightarrow 0$.

In the case $\omega_{n} \rightarrow \omega>0$, by Lemmas 2.3 and 2.6, we obtain

Lemma 3.2 Let $\left(\omega_{n}\right) \subset(0,+\infty)$ and $\left(u_{n}\right) \subset X$ be such that $\omega_{n} \rightarrow \omega>0$, $\varphi_{\omega_{n}}\left(u_{n}\right) \rightarrow l \in \mathbf{R}$ and $\varphi_{\omega_{n}}^{\prime}\left(u_{n}\right) \rightarrow 0$. If $\left(T\left(u_{n}\right)\right)$ is bounded then there exists $u \in \mathcal{K}_{\omega}$ such that, up to a subsequence, $u_{n} \rightarrow u$ weakly in $X, T\left(u_{n}\right) \rightarrow T(u)$, $\varphi_{\omega_{n}}\left(u_{n}-u\right) \rightarrow l-\varphi_{\omega}(u)$ and $\varphi_{\omega_{n}}^{\prime}\left(u_{n}-u\right) \rightarrow 0$.

While, in the case $\omega_{n} \rightarrow 0$, we have the following characterization

Lemma 3.3 Let $\left(\omega_{n}\right) \subset(0,+\infty)$ and $\left(u_{n}\right) \subset X$ be such that $\omega_{n} \rightarrow 0$, $\varphi\left(u_{n}\right) \rightarrow l \in \mathbf{R}, \varphi_{\omega_{n}}^{\prime}\left(u_{n}\right) \rightarrow 0$ and $\omega_{n} T\left(u_{n}\right) \rightarrow r \in \mathbf{R}$. Then there exists $u \in X \backslash\{0\}$ such that, up to a subsequence, there results:

(i) $u_{n}\left(\cdot+T\left(u_{n}\right)\right) \rightarrow u$ weakly in $X$ and $\psi_{b \beta}^{\prime}(u)=0$, where $a\left(\cdot+T\left(u_{n}\right)\right) \rightarrow$ $b \in H(a)$ and $\alpha\left(\omega_{n}\left(\cdot+T\left(u_{n}\right)\right)\right) \rightarrow \alpha(r)=\beta$ in $L_{l o c}^{\infty}$,

(ii) $\varphi_{\omega_{n}}\left(u_{n}-u\left(\cdot-T\left(u_{n}\right)\right)\right) \rightarrow l-\psi_{b \beta}(u)$ and $\varphi_{\omega_{n}}^{\prime}\left(u_{n}-u\left(\cdot-T\left(u_{n}\right)\right)\right) \rightarrow 0$. 
Proof. By $(2.2),\left(u_{n}\right)$ is bounded in $X$. Then, setting $v_{n}=u_{n}\left(\cdot+T\left(u_{n}\right)\right)$, there exists $u \in X$ such that, up to a subsequence, $v_{n} \rightarrow u$ weakly in $X$. By Lemma 2.5 and Remark 2.4, we have $\liminf _{n \rightarrow \infty}\left|u_{n}\left(T\left(u_{n}\right)\right)\right| \geq \delta>0$. Therefore, since $v_{n} \rightarrow u$ in $L_{l o c}^{\infty}$, we obtain $u \not \equiv 0$.

By the Bochner's criterion (see e.g. [Be]), up to a subsequence we have $a\left(\cdot+T\left(u_{n}\right)\right) \rightarrow b \in H(a)$ in $L^{\infty}$. Moreover, since $\omega_{n} \rightarrow 0$, we obtain $\alpha\left(\omega_{n}(t+\right.$ $\left.\left.T\left(u_{n}\right)\right)\right) \rightarrow \beta=\alpha(r)$ in $L_{l o c}^{\infty}$. Then, for any $h \in C_{c}^{\infty}\left(\mathbf{R}, \mathbf{R}^{N}\right)$, since $v_{n} \rightarrow u$ strongly in $L_{l o c}^{\infty}$ and $\varphi_{\omega_{n}}^{\prime}\left(u_{n}\right) \rightarrow 0$, we obtain

$$
\psi_{b \beta}^{\prime}(u) h=\varphi_{\omega_{n}}^{\prime}\left(u_{n}\right) h\left(\cdot-T\left(u_{n}\right)\right)+o(1)=o(1) .
$$

By density, this implies that $\psi_{b \beta}^{\prime}(u)=0$. Then $(i)$ holds.

To prove $(i i)$, first note that, since $v_{n} \rightarrow u$ weakly in $X$, we have

$$
\begin{gathered}
\int_{\mathbf{R}}\left|W\left(v_{n}-u\right)-W\left(v_{n}\right)+W(u)\right| d t \rightarrow 0, \\
\sup _{h \in X,\|h\|=1}\left[\int_{\mathbf{R}}\left|W^{\prime}\left(v_{n}-u\right)-W^{\prime}\left(v_{n}\right)+W^{\prime}(u)\right||h| d t\right] \rightarrow 0 .
\end{gathered}
$$

Therefore

$$
\begin{array}{r}
\varphi_{\omega_{n}}\left(u_{n}-u\left(\cdot-T\left(u_{n}\right)\right)\right)+\varphi_{\omega_{n}}\left(u\left(\cdot-T\left(u_{n}\right)\right)\right)-\varphi_{\omega_{n}}\left(u_{n}\right) \rightarrow 0, \\
\varphi_{\omega_{n}}^{\prime}\left(u_{n}-u\left(\cdot-T\left(u_{n}\right)\right)\right)+\varphi_{\omega_{n}}^{\prime}\left(u\left(\cdot-T\left(u_{n}\right)\right)\right)-\varphi_{\omega_{n}}^{\prime}\left(u_{n}\right) \rightarrow 0 .
\end{array}
$$

Since $\varphi_{\omega_{n}}\left(u_{n}\right) \rightarrow l$ and $\varphi_{\omega_{n}}^{\prime}\left(u_{n}\right) \rightarrow 0$, we obtain

$$
\begin{aligned}
& \varphi_{\omega_{n}}\left(u_{n}-u\left(\cdot-T\left(u_{n}\right)\right)\right)+\varphi_{\omega_{n}}\left(u\left(\cdot-T\left(u_{n}\right)\right)\right) \rightarrow l, \\
& \varphi_{\omega_{n}}^{\prime}\left(u_{n}-u\left(\cdot-T\left(u_{n}\right)\right)\right)+\varphi_{\omega_{n}}^{\prime}\left(u\left(\cdot-T\left(u_{n}\right)\right)\right) \rightarrow 0 .
\end{aligned}
$$

Finally, since $\left|b(t)-a\left(t+T\left(u_{n}\right)\right)+\beta-\alpha\left(\omega_{n}\left(t+T\left(u_{n}\right)\right)\right)\right| \rightarrow 0$ in $L_{l o c}^{\infty}$, we obtain $\varphi_{\omega_{n}}\left(u\left(\cdot-T\left(u_{n}\right)\right)\right) \rightarrow \psi_{b \beta}(u)$ and $\varphi_{\omega_{n}}^{\prime}\left(u\left(\cdot-T\left(u_{n}\right)\right)\right) \rightarrow \psi_{b \beta}^{\prime}(u)=0$ and therefore the lemma follows.

We are now interested in compactness results for sequences $\left(u_{n}\right) \subset X$ such that $\left(\varphi_{\omega_{n}}\left(u_{n}\right)\right)$ is bounded, $\varphi_{\omega_{n}}^{\prime}\left(u_{n}\right) \rightarrow 0$ and $\left(\omega_{n} T\left(u_{n}\right)\right) \subset[-\bar{r}, \bar{r}]$ for some sequence $\left(\omega_{n}\right) \subset(0,+\infty)$. First we have the following level estimate

Lemma 3.4 For every $h>0$ there exists $\omega_{h}>0$ such that for all $\left(\omega_{n}\right) \subset$ $\left(0, \omega_{h}\right]$ and $\left(u_{n}\right) \subset X$ with $\varphi_{\omega_{n}}^{\prime}\left(u_{n}\right) \rightarrow 0$ and $\left|T\left(u_{n}\right)\right| \leq \frac{\bar{r}}{\omega_{n}}$ we have $c_{\bar{\alpha}}-h \leq$ $\liminf \varphi_{\omega_{n}}\left(u_{n}\right)$. 
Proof. Indeed, if not, using a diagonal procedure, we obtain that there exists $h>0$, a sequence $\left(\omega_{n}\right) \subset(0,+\infty)$ with $\omega_{n} \rightarrow 0$ and a sequence $\left(u_{n}\right) \subset X$ such that $\omega_{n}\left|T\left(u_{n}\right)\right| \leq \bar{r}, \varphi_{\omega_{n}}^{\prime}\left(u_{n}\right) \rightarrow 0$ and $\liminf \varphi_{\omega_{n}}\left(u_{n}\right) \leq c_{\bar{\alpha}}-h$. Up to a subsequence, we have that $\omega_{n} T\left(u_{n}\right) \rightarrow r \in[-\bar{r}, \bar{r}]$ and $\varphi_{\omega_{n}}\left(u_{n}\right) \rightarrow l \leq c_{\bar{\alpha}}-h$. By Lemma 3.3 and the choice of $\bar{r}$, there exists $u \in X \backslash\{0\}$ such that, setting $\lim a\left(\cdot+T\left(u_{n}\right)\right)=b \in H(a)$ and $\lim \alpha\left(\omega_{n}\left(\cdot+T\left(u_{n}\right)\right)\right)=\alpha(r)=$ $\beta \leq \bar{\alpha}$, there results $\psi_{b \beta}^{\prime}(u)=0, \varphi_{\omega_{n}}\left(u_{n}-u\left(\cdot-T\left(u_{n}\right)\right)\right) \rightarrow l-\psi_{b \beta}(u)$ and $\varphi_{\omega_{n}}^{\prime}\left(u_{n}-u\left(\cdot-T\left(u_{n}\right)\right)\right) \rightarrow 0$. Then, by Lemma 3.1, $l-\psi_{b \beta}(u) \geq 0$ and therefore, by Remark 2.2, $c_{\bar{\alpha}}-h \geq l \geq \psi_{b \beta}(u) \geq c_{\beta} \geq c_{\bar{\alpha}}$, a contradiction.

The following compactness result holds

Lemma 3.5 There exist $h_{0}>0$ and $\omega_{0}>0$ such that if $\left(\omega_{n}\right) \subset\left(0, \omega_{0}\right)$ and $\left(u_{n}\right) \subset X$ satisfy $\varphi_{\omega_{n}}\left(u_{n}\right) \rightarrow l \leq c_{\bar{\alpha}}+h_{0}, \varphi_{\omega_{n}}^{\prime}\left(u_{n}\right) \rightarrow 0$ and $\left|T\left(u_{n}\right)\right| \leq \frac{\bar{r}}{\omega_{n}}$ then $\left(u_{n}\left(\cdot+T\left(u_{n}\right)\right)\right.$ is precompact in $X$.

Proof. Let $h_{0} \in\left(0, \frac{\bar{\lambda}}{2}\right)$, where $\bar{\lambda}$ is given by Remark 2.1 and $\omega_{0}=\omega_{h_{0}}$ by Lemma 3.4. Let $\left(\omega_{n}\right) \subset\left(0, \omega_{0}\right)$ and $\left(u_{n}\right) \subset X$ satisfying the statement of the lemma. Then, up to a subsequence, two cases may occur: $(i) \omega_{n} \rightarrow 0$, or $(i i)$ $\omega_{n} \rightarrow \omega \in\left(0, \omega_{0}\right]$.

In the case $(i)$, by Lemma 3.3, there exists $u \in X \backslash\{0\}$ such that $u_{n}(\cdot+$ $\left.T\left(u_{n}\right)\right) \rightarrow u$ weakly in $X$. Moreover, setting $\lim a\left(\cdot+T\left(u_{n}\right)\right)=b \in H(a)$ and $\lim \alpha\left(\omega_{n}\left(\cdot+T\left(u_{n}\right)\right)\right)=\beta$, there results $\beta \leq \bar{\alpha}, \psi_{b \beta}^{\prime}(u)=0, \varphi_{\omega_{n}}\left(u_{n}-\right.$ $\left.u\left(\cdot-T\left(u_{n}\right)\right)\right) \rightarrow l-\psi_{b \beta}(u)$ and $\varphi_{\omega_{n}}^{\prime}\left(u_{n}-u\left(\cdot-T\left(u_{n}\right)\right)\right) \rightarrow 0$. Then, since by Remarks 2.2 and $3.1 \psi_{b \beta}(u) \geq c_{\beta} \geq c_{\bar{\alpha}}$, we have $l-\psi_{b \beta}(u) \leq l-c_{\bar{\alpha}} \leq h_{0}<\frac{\bar{\lambda}}{2}$. By Lemma 3.1, we obtain $u_{n}-u\left(\cdot-T\left(u_{n}\right)\right) \rightarrow 0$, i.e., $u_{n}\left(\cdot+T\left(u_{n}\right)\right) \rightarrow u$ strongly in $X$ and the lemma is proved in this case.

In the case $(i i)$, by Lemma 3.2 , we have that $u_{n} \rightarrow u \in \mathcal{K}_{\omega}$ weakly in $X$, $\varphi_{\omega_{n}}\left(u_{n}-u\right) \rightarrow l-\varphi_{\omega}(u), \varphi_{\omega_{n}}^{\prime}\left(u_{n}-u\right) \rightarrow 0$ and $T\left(u_{n}\right) \rightarrow T(u)$. Therefore, by Lemma 3.4 in particular we have $\varphi_{\omega}(u) \geq c_{\bar{\alpha}}-h_{0}$. Then $l-\varphi_{\omega}(u) \leq$ $l-\left(c_{\bar{\alpha}}-h_{0}\right) \leq 2 h_{0}<\bar{\lambda}$ and hence, by Lemma $3.1, u_{n} \rightarrow u$ strongly in $X$ and $u_{n}\left(\cdot+T\left(u_{n}\right)\right) \rightarrow u(\cdot+T(u))$.

In particular it follows

Lemma 3.6 There exist $\nu_{0} \in\left(0, \frac{\bar{\rho}}{2}\right)$ and $R_{0}>0$ such that if $u \in X$ with $\varphi_{\omega}(u) \leq c_{\bar{\alpha}}+h_{0},\left\|\varphi_{\omega}^{\prime}(u)\right\| \leq \nu_{0}$ and $|T(u)| \leq \frac{\bar{r}}{\omega}$ for some $\omega \in\left(0, \omega_{0}\right)$ then

$$
\|u\|_{|t-T(u)| \geq R_{0}}<\frac{\bar{\rho}}{2}
$$


Proof. Arguing by contradiction suppose that there exist $R_{n} \rightarrow+\infty,\left(\omega_{n}\right) \subset$ $\left(0, \omega_{0}\right)$ and $\left(u_{n}\right) \subset X$ such that $\lim \sup \varphi_{\omega_{n}}\left(u_{n}\right) \leq c_{\bar{\alpha}}+h_{0}, \varphi_{\omega_{n}}^{\prime}\left(u_{n}\right) \rightarrow$ $0,\left|T\left(u_{n}\right)\right| \leq \frac{\bar{r}}{\omega_{n}}$ and $\left\|u_{n}\right\|_{\left|t-T\left(u_{n}\right)\right| \geq R_{n}} \geq \frac{\bar{\rho}}{2}$. This is impossible since by Lemma 3.5 the sequence $\left(u_{n}\left(\cdot+T\left(u_{n}\right)\right)\right)$ is precompact in $X$ and therefore $\left\|u_{n}\left(\cdot+T\left(u_{n}\right)\right)\right\|_{|t| \geq R_{n}} \rightarrow 0$ as $n \rightarrow \infty$.

Then we obtain

Lemma 3.7 There exist $\bar{h} \in\left(0, h_{0}\right), \bar{\nu} \in\left(0, \nu_{0}\right)$ and $\bar{\omega} \in\left(0, \omega_{0}\right)$ such that if $u \in X$ is such that $\varphi_{\omega}(u) \leq c_{\bar{\alpha}}+\bar{h},\left\|\varphi_{\omega}^{\prime}(u)\right\| \leq \bar{\nu}$ and $|T(u)| \leq \frac{\bar{r}}{\omega}$ for some $\omega \in(0, \bar{\omega})$ then

$$
\|u\|_{|t| \geq \frac{\tilde{r}}{\omega}}<\frac{\bar{\rho}}{2}
$$

Proof. By contradiction suppose that there exist $h_{n} \rightarrow 0, \nu_{n} \rightarrow 0, \omega_{n} \rightarrow 0$ and $\left(u_{n}\right) \subset X$ such that $\varphi_{\omega_{n}}\left(u_{n}\right) \leq c_{\bar{\alpha}}+h_{n},\left\|\varphi_{\omega_{n}}^{\prime}\left(u_{n}\right)\right\| \leq \nu_{n},\left|T\left(u_{n}\right)\right| \leq \frac{\bar{r}}{\omega_{n}}$ and $\left\|u_{n}\right\|_{|t| \geq \frac{\tilde{r}}{\omega_{n}}} \geq \frac{\rho}{2}$. Then, by Lemma 3.6 we have that $\frac{\tilde{r}}{\omega_{n}}-R_{0} \leq\left|T\left(u_{n}\right)\right| \leq$ $\frac{\bar{r}}{\omega_{n}}$ and therefore, up to a subsequence, $\left|T\left(u_{n}\right) \omega_{n}\right| \rightarrow r \in[\tilde{r}, \bar{r}]$. Then, by the choice of $\tilde{r}$, we obtain that $\alpha\left(\omega_{n}\left(t+T\left(u_{n}\right)\right)\right) \rightarrow \alpha(r)=\beta \leq \underline{\alpha}<\bar{\alpha}$ in $L_{l o c}^{\infty}$. Since $\lim \sup \varphi_{\omega_{n}}\left(u_{n}\right) \leq c_{\bar{\alpha}}, \varphi_{\omega_{n}}^{\prime}\left(u_{n}\right) \rightarrow 0$ and $\left|T\left(u_{n}\right)\right| \leq \frac{\bar{r}}{\omega_{n}}$, by Lemma 3.3 we obtain that, up to a subsequence, $\left.u_{n}\left(\cdot+T\left(u_{n}\right)\right)\right) \rightarrow u \in X \backslash\{0\}$ weakly in $X, \psi_{b \beta}^{\prime}(u)=0$ and, by Lemma $3.2, \psi_{b \beta}(u) \leq c_{\bar{\alpha}}$ for some $b \in H(a)$. While, by Remark 3.1, we have $\psi_{b \beta}(u) \geq c_{\beta} \geq c_{\underline{\alpha}}>c_{\bar{\alpha}}$, a contradiction.

Finally we have

Lemma 3.8 If $u \in X$ is such that $\varphi_{\omega}(u) \leq c_{\bar{\alpha}}+\bar{h}$ and $\frac{\tilde{r}}{\omega} \leq|T(u)| \leq \frac{\bar{r}}{\omega}$ for some $\omega \in(0, \bar{\omega})$ then $\left\|\varphi_{\omega}^{\prime}(u)\right\| \geq \bar{\nu}$. In particular for all $\omega \in(0, \bar{\omega})$ we have

$$
T\left(\mathcal{K}_{\omega} \cap\left\{\varphi_{\omega} \leq c_{\bar{\alpha}}+\bar{h}\right\}\right) \cap\left\{t \in \mathbf{R}\left|\frac{\tilde{r}}{\omega} \leq\right| t \mid \leq \frac{\bar{r}}{\omega}\right\}=\emptyset .
$$

Proof. Indeed, if there exists $u \in X$ such that $\varphi_{\omega}(u) \leq c_{\bar{\alpha}}+\bar{h}, \frac{\tilde{r}}{\omega} \leq$ $|T(u)| \leq \frac{\bar{r}}{\omega}$ and $\left\|\varphi_{\omega}^{\prime}(u)\right\|<\bar{\nu}$ for some $\omega \in(0, \bar{\omega})$ then, by Lemma 3.7, $\|u\|_{|t| \geq \frac{\tilde{r}}{\omega}}<\frac{\bar{\rho}}{2}$. Therefore, if $T(u) \geq \frac{\tilde{r}}{\omega}$ we obtain a contradiction since, by definition, $\bar{\rho}=\|u\|_{t \geq T(u)} \leq\|u\|_{t \geq \frac{\tilde{r}}{\omega}}<\frac{\bar{\rho}}{2}$. In the other case, $T(u) \leq-\frac{\tilde{r}}{\omega}$, we obtain $\|u\|^{2} \leq\|u\|_{t \geq T(u)}^{2}+\|u\|_{t \leq-\frac{\tilde{r}}{\omega}}^{2}<2 \bar{\rho}^{2}$ and, by the choice of $\bar{\rho}$, we have $\left\|\varphi^{\prime}(u)\right\| \geq \frac{3}{4}\|u\| \geq \nu$, since $\nu<\frac{\overline{\bar{\rho}}}{2}$, contrary to the assumption.

Now, to prove Theorem 3.1, by Lemma 3.8, it is sufficient to show that $c_{\omega}<c_{\bar{\alpha}}+\bar{h}$ for all $\omega$ small enough. In fact, we have 
Lemma 3.9 There results $\lim \sup _{\omega \rightarrow 0} c_{\omega} \leq c_{\bar{\alpha}}$. In particular, there exists $\tilde{\omega} \in(0, \bar{\omega})$ such that $c_{\omega}<c_{\bar{\alpha}}+\bar{h}$ for all $\omega \in(0, \tilde{\omega})$.

Proof. First note that setting $\varphi_{b \omega}(u)=\frac{1}{2}\|u\|^{2}-\int_{\mathbf{R}}(b(t)+\alpha(\omega t)) W(u) d t$, like in Remark 3.1, we have that $c_{\omega}$ is the mountain pass level of $\varphi_{b \omega}$ for any $b \in H(a)$. Moreover we know that there exist $b \in H(a)$ and $\gamma \in \Gamma$ such that $\max _{s \in[0,1]} \psi_{b \bar{\alpha}}(\gamma(s))=c_{\bar{\alpha}}$. Since $\gamma([0,1])$ is compact, by $\left(W_{2}\right)$, for all $h>0$ there exists $\delta_{h} \in(0, \bar{\delta})$ such that

$$
\int_{\left\{t \in \mathbf{R}|| \gamma(s)(t) \mid \leq \delta_{h}\right\}} W(\gamma(s)) d t \leq \frac{h}{4 \bar{a}}
$$

for all $s \in[0,1]$. In addition, there exists $R_{h}>0$ such that for all $s \in[0,1]$ $\sup _{|t| \geq R_{h}}|\gamma(s)(t)| \leq \delta_{h}$. Therefore for all $s \in[0,1]$ we obtain

$$
\begin{aligned}
& \left|\varphi_{b \omega}(\gamma(s))-\psi_{b \bar{\alpha}}(\gamma(s))\right| \\
& \leq \int_{|t| \geq R_{h}}|\bar{\alpha}-\alpha(\omega t)| W(\gamma(s)) d t+\int_{|t| \leq R_{h}}|\bar{\alpha}-\alpha(\omega t)| W(\gamma(s)) d t \\
& \leq \frac{h}{2}+\sup _{|t| \leq R_{h}}|\bar{\alpha}-\alpha(\omega t)| \int_{\mathbf{R}} W(\gamma(s)) d t .
\end{aligned}
$$

Then, since $\alpha(\omega t) \rightarrow \bar{\alpha}$ in $L_{l o c}^{\infty}$ as $\omega \rightarrow 0$ and $\gamma([0,1])$ is compact, we have $\limsup _{\omega \rightarrow 0} \sup _{s \in[0,1]}\left|\varphi_{b \omega}(\gamma(s))-\psi_{b \bar{\alpha}}(\gamma(s))\right| \leq h$ for any $h>0$ and hence $\sup _{s \in[0,1]}\left|\varphi_{b \omega}(\gamma(s))-\psi_{b \bar{\alpha}}(\gamma(s))\right|=o(1)$ as $\omega \rightarrow 0$. In particular, $c_{\omega} \leq \max _{s \in[0,1]} \varphi_{b \omega}(\gamma(s)) \leq \max _{s \in[0,1]} \psi_{b \bar{\alpha}}(\gamma(s))+o(1)=c_{\bar{\alpha}}+o(1)$ as $\omega \rightarrow 0$.

\section{Multibump solutions}

In this section we consider the system $\left(D_{a}\right)$ for a given positive almost periodic function $a$ and we discuss a discreteness assumption on the set of homoclinic solutions of $\left(D_{a}\right)$, already studied in [MNT1], [MNT2]. Precisely, letting $c_{a}$ be the mountain pass level of the functional $\varphi_{a}(u)=\frac{1}{2}\|u\|^{2}-$ $\int_{\mathbf{R}} a(t) W(u(t)) d t$, we assume that

$$
\text { there exists } \tilde{c}>c_{a} \text { such that } T\left(\mathcal{K}_{a} \cap\left\{\varphi_{a} \leq \tilde{c}\right\}\right) \neq \mathbf{R}
$$

where the function $T$ has been introduced in Section 2 and $\mathcal{K}_{a}=\{u \in$ $\left.X \mid \varphi_{a}^{\prime}(u)=0, u \neq 0\right\}$. 
Here below, we outline how the assumption $(H)$ togheter with the regularity of $\varphi_{a}$ leads to the existence of a compact set $K_{0} \subset \mathcal{K}_{a}$ that satisfies stability properties suitable to apply the Séré product minimax and then to prove that $\left(D_{a}\right)$ admits a multibump dynamics. We omit the proofs that can be obtained by adapting the arguments already developped in [MNT1] and [MNT2].

We explicitely remark that the functional $\varphi_{\omega}$ defined in (3.1) verifies the assumption $(H)$ whenever $\omega>0$ is sufficiently small (condition $\left(H_{\omega}\right)$ in Theorem 3.1) and therefore that Theorem 1.1 follows by the following construction. Moreover, we note that if $\varphi_{a}$ verifies $(H)$ then also $\varphi_{a+h}$ satisfies it whenever $h \in C(\mathbf{R}, \mathbf{R})$ has $L^{\infty}$ norm sufficiently small. By Theorem 1.1, this implies Theorem 1.2.

By $(H)$, let $t_{0} \in \mathbf{R} \backslash T\left(\mathcal{K}_{a} \cap\left\{\varphi_{a} \leq \tilde{c}\right\}\right)$. By Lemma 2.6 we plainly derive that $\exists l_{0}, \nu_{0}>0$ such that

$$
\text { if } T(u) \in I_{0}=\left[t_{0}-l_{0}, t_{0}+l_{0}\right] \text { and } \varphi_{a}(u) \leq \tilde{c} \text { then }\left\|\varphi_{a}^{\prime}(u)\right\| \geq \nu_{0} .
$$

By (4.1) and the almost periodicity of $a$, setting $I_{\tau}=-\tau+I_{0}$, we obtain

Lemma 4.1 There exists $\varepsilon_{0}>0$ such that if $T(u) \in I_{\tau}$ for some $\tau \in P_{\varepsilon_{0}}(a)$ and $\varphi_{a}(u) \leq c_{a}+\frac{\tilde{c}-c_{a}}{2}$ then $\left\|\varphi_{a}^{\prime}(u)\right\| \geq \frac{\nu_{0}}{2}$.

Let $\left(\tau_{j}\right)_{j \in \mathbf{Z}} \subset P_{\varepsilon_{0}}(a)$ be such that $\tau_{j}-\tau_{j-1}>2 l_{0} \forall j \in \mathbf{Z}$. We denote $\bar{\nu}=\frac{1}{4} \min \left\{\nu_{0}, \bar{\rho}\right\}, h_{0}=\frac{\tilde{c}-c_{a}}{2}, J_{j}=\left(-\tau_{j+1}+t_{0}+l_{0},-\tau_{j}+t_{0}-l_{0}\right)$ and $A_{j}=\left\{u \in X \mid\left\|\varphi_{a}^{\prime}(u)\right\| \leq \bar{\nu}, \varphi_{a}(u) \leq c_{a}+h_{0}\right.$ and $\left.T(u) \in J_{j}\right\}$. By Lemma 4.1 we have

$$
\left\{\left\|\varphi_{a}^{\prime}\right\| \leq \bar{\nu}\right\} \cap\left\{\varphi_{a} \leq c_{a}+h_{0}\right\} \backslash B_{\bar{\rho}}(0)=\cup_{j \in \mathbf{Z}} A_{j} .
$$

Moreover, by Lemma 2.7, we obtain

Lemma 4.2 There exists $r_{0}>0$ such that if $i \neq j$ then $\operatorname{dist}\left(A_{i}, A_{j}\right) \geq r_{0}$.

By a classical pseudogradient construction one can prove the existence of a locally Lipschitz continuous vector field $V: X \rightarrow X$ which verifies $\|V(u)\| \leq 1, \varphi_{a}^{\prime}(u) V(u) \leq 0$ for any $u \in X, V(u)=0$ for any $u \in\left\{\varphi_{a} \leq 0\right\}$ and moreover, by Lemma 4.1, $\varphi_{a}^{\prime}(u) V(u) \leq-\frac{1}{2} \bar{\nu}$ for any $u \in\left\{\varphi_{a} \leq c_{a}+\right.$ $\left.h_{0}\right\} \backslash\left(B_{\bar{\rho}}(0) \cup \cup_{j \in \mathbf{Z}} A_{j}\right)$. Let $\eta \in C\left(\mathbf{R}^{+} \times X, X\right)$ be the flow associated to the Cauchy problem

$$
\left\{\begin{array}{l}
\frac{d}{d s} \eta(s, u)=V(\eta(s, u)), \quad s>0 \\
\eta(0, u)=u .
\end{array}\right.
$$

Then by the properties of $V$ we obtain (see e.g. [MNT1] for more details) 
Lemma 4.3 Let $\bar{r} \in\left(0, \frac{r_{0}}{3}\right)$, there exist $\bar{h} \in\left(0, h_{0}\right)$ and $\bar{s}>0$ such that

$$
\eta\left(\bar{s},\left\{\varphi_{a} \leq c_{a}+\bar{h}\right\}\right) \subset\left\{\varphi_{a} \leq c_{a}-\bar{h}\right\} \cup \cup_{j \in \mathbf{Z}} B_{\bar{r}}\left(A_{j}\right) .
$$

By Lemma 4.3, deforming a path in $\Gamma$ contained in $\left\{\varphi_{a} \leq c_{a}+\bar{h}\right\}$, we obtain the existence of a path $\gamma \in \Gamma$ and, by compactness, a finite number of sets $A_{i_{1}}, \ldots, A_{i_{k}}$ for which

(i) $\max _{s \in[0,1]} \varphi_{a}(\gamma(s)) \leq c_{a}+\bar{h}$;

(ii) if $\gamma(s) \notin \cup_{p=1}^{k} B_{\bar{r}}\left(A_{i_{p}}\right)$ then $\varphi_{a}(\gamma(s)) \leq c_{a}-\bar{h}$.

Then, by Lemma 4.2 and the definition of the mountain pass level $c_{a}$, there exists $p \in\{1, \ldots k\}$ such that, setting $A_{0}=A_{i_{p}}$ and $\Omega_{0}=B_{\bar{r}}\left(A_{0}\right) \cap\left\{\varphi_{a} \leq\right.$ $\left.c_{a}+\bar{h}\right\}$, there exist $s_{1}, s_{2} \in[0,1]$ for which $u_{0}=\gamma\left(s_{1}\right), u_{1}=\gamma\left(s_{2}\right) \in$ $\partial B_{\bar{r}}\left(A_{0}\right) \cap\left\{\varphi_{a} \leq c_{a}-\bar{h}\right\}, \gamma(s) \in \Omega_{0}$ for any $s \in\left(s_{1}, s_{2}\right)$ and $u_{0}, u_{1}$ are not path connectible in $\left\{\varphi_{a}<c_{a}\right\}$. Then, considered the class

$\bar{\Gamma}=\left\{\gamma \in C([0,1], X) \mid \gamma(0)=u_{0}, \gamma(1)=u_{1}, \gamma([0,1]) \subset \Omega_{0} \cup\left\{\varphi_{a} \leq c_{a}-\frac{\bar{h}}{2}\right\}\right\}$,

we have $\bar{\Gamma} \neq \emptyset$ and, putting $\bar{c}=\inf _{\gamma \in \bar{\Gamma}} \max _{s \in[0,1]} \varphi_{a}(\gamma(s))$, we obtain $c_{a} \leq$ $\bar{c} \leq c_{a}+\bar{h}<\tilde{c}$.

Let $K_{0}=\mathcal{K}_{a} \cap A_{0}$. Since, by Corollary 2.1, the PS condition holds in $\Omega_{0}$, with arguments similar to the ones used in [MNT1], it is possible to prove the existence of a pseudogradient flow which leaves the class $\bar{\Gamma}$ invariant and then to prove the following result

Lemma 4.4 $K_{0} \neq \emptyset$ and for any $r \in\left(0, \frac{\bar{r}}{4}\right)$ there exists $\nu_{r}>0$ such that

$$
\left\|\varphi_{a}^{\prime}(u)\right\| \geq \nu_{r} \text { for any } u \in \Omega_{0} \backslash B_{\frac{r}{4}}\left(K_{0}\right) .
$$

Moreover there exists $h_{r} \in\left(0, \frac{\bar{h}}{2}\right)$ such that for any $h>0$ there exists $\gamma \in \bar{\Gamma}$ for which

(i) $\max _{s \in[0,1]} \varphi_{a}(\gamma(s)) \leq \bar{c}+h$;

(ii) $\gamma([0,1]) \subset B_{\frac{r}{2}}\left(K_{0}\right) \cup\left\{\varphi_{a} \leq \bar{c}-h_{r}\right\}$,

(iii) $\operatorname{supp} \gamma(s) \subset[-R, R]$ for any $s \in[0,1]$, where $R$ is a positive constant independent of $s$. 
Finally, since $\varphi_{a} \in C^{2 N}(X, \mathbf{R})$, applying a refined version of the SardSmale Lemma (see [B, Lemma 3.1.47]), as in [MNT2] we obtain the following property

Lemma $4.5[0, \tilde{c}] \backslash \varphi_{a}\left(K_{0}\right)$ is open and dense in $[0, \tilde{c}]$.

By Lemmas 4.4, 4.5 and the almost periodicity of $a$ we can characterize also the sets $K_{\tau}=\left\{u(\cdot-\tau) \mid u \in K_{0}\right\}$ in $\Omega_{\tau}=\left\{u(\cdot-\tau) \mid u \in \Omega_{0}\right\}$, whenever $\tau \in P_{\varepsilon}(a)$, for $\varepsilon$ small enough.

Lemma 4.6 For all $r \in\left(0, \frac{\bar{r}}{4}\right)$ and $h \in\left(0, \frac{h_{r}}{8}\right)$, let $\nu_{r}>0$ and $\gamma \in \bar{\Gamma}$ be given according to Lemma 4.4. Then there exist $\left[l_{1}^{+}, l_{2}^{+}\right] \subset\left(\bar{c}+\frac{3}{2} h, \bar{c}+2 h\right)$, $\left[l_{1}^{-}, l_{2}^{-}\right] \subset\left(\bar{c}-h, \bar{c}-\frac{1}{2} h\right), \mu>0$ and $\bar{\varepsilon}>0$ such that for any $\tau \in P_{\bar{\varepsilon}}(a)$ we have

Annuli Property : $u \in \Omega_{\tau} \cap\left\{\varphi_{a}<\bar{c}+2 h\right\} \backslash B_{\frac{r}{4}}\left(K_{\tau}\right) \Rightarrow\left\|\varphi_{a}^{\prime}(u)\right\| \geq \nu_{r}$,

Slices Property : $u \in \Omega_{\tau} \cap\left\{l_{1}^{ \pm} \leq \varphi_{a} \leq l_{2}^{ \pm}\right\} \Rightarrow\left\|\varphi_{a}^{\prime}(u)\right\| \geq \mu$,

Topological Property: setting $\gamma_{\tau}(s)=\gamma(s)(\cdot-\tau)$ we have

(i) $\gamma_{\tau}(0), \gamma_{\tau}(1) \in \partial \Omega_{\tau}$ are not connectible in $\Omega_{\tau} \cap\left\{\varphi_{a}<\bar{c}-\frac{h}{2}\right\}$;

(ii) $\gamma_{\tau}([0,1]) \subset\left\{\varphi_{a} \leq \bar{c}+\frac{3}{2} h\right\}$;

(iii) $\gamma_{\tau}([0,1]) \subset B_{\frac{r}{2}}\left(K_{\tau}\right) \cup\left\{\varphi_{a} \leq \bar{c}-\frac{h_{r}}{4}\right\}$;

(iv) $\exists R>0$ such that $\operatorname{supp} \gamma_{\tau}(s) \subset[-R+\tau, R+\tau]$ for any $s \in[0,1]$.

It is nowadays well known that these properties are sufficient to apply the Séré multibump construction, [S2], and then to prove the existence of multibump solution for the systems $\left(D_{\alpha}\right)$ (see [MNT1] and [MNT2]).

Theorem 4.1 If $(H)$ is satisfied then for any $r>0$ there is $N_{r}>0$ and $\varepsilon_{r}>0$ such that for any sequence $\left(p_{j}\right) \subset P_{\varepsilon_{r}}(a)$, with $p_{j+1}-p_{j} \geq N_{r}$ and for any $\sigma=\left(\sigma_{j}\right) \in\{0,1\}^{\mathbf{Z}}$ there exists a solution $v_{\sigma}$ of $\left(D_{a}\right)$ verifying

$$
\inf _{u \in K_{0}}\left\|v_{\sigma}-\sigma_{j} u\left(\cdot-p_{j}\right)\right\|_{C^{1}\left(I_{j}\right)}<r \quad \forall j \in \mathbf{Z},
$$

where $I_{j}=\left[\frac{p_{j-1}+p_{j}}{2}, \frac{p_{j}+p_{j+1}}{2}\right]$. In addition $v_{\sigma}$ is a homoclinic solution whenever $\sigma_{j}=0$ definitively. 


\section{References}

[AM] F. Alessio and P. Montecchiari, Multibump solutions for a class of Lagrangian systems slowly oscillating at infinity, preprint (1996).

[AB] A. Ambrosetti and M. Badiale, Homoclinics: Poincarè-Melnikov type results via a variational approach, C.R. Acad. Sci. Paris, t.323, Série I (1996), 753-758; and Ann. Inst. H. Poincaré, Anal. Non Linéaire, to appear.

[ABC] A. Ambrosetti, M. Badiale and S. Cingolani, Semiclassical states of nonlinear Schrödinger equation, Arch. Rat. Mech. Anal., to appear.

[An] S. Angenent, A variational interpretation of Melnikov's function and exponentially small separatrix splitting, Lecture notes of the London Math. Soc, Symplectic geometry, ed. Dietmar Salamon.

[BG] V. Benci and F. Giannoni, Homoclinic orbits on compact manifolds, J. Math. Anal. Appl. 157 (1991), 568-576.

[B] M.S. Berger, Nonlinearity and Functional Analysis, Lectures on Nonlinear Problems in Mathematical Analysis, Academic Press (1977).

[Be] A.S. Besicovitch, Almost Periodic Functions, Dover Pubblications Inc. (1954).

[Be1] U. Bessi, A Variational Proof of a Sitnikov-like Theorem, Nonlinear Anal. TMA 20 (1993), 1303-1318.

[Be2] U. Bessi, Global Homoclinic Bifurcation for Damped Systems, Math. Z. 218 (1995), 387-415.

[Be3] U. Bessi, Homoclinic and Period-doubling Bifurcations for Damped Systems, Ann. Inst. H. Poincaré, Anal. Non Linéaire 12 (1995), 1-25.

[BBo] M. Berti and P. Bolle, Homoclinics and chaotic behaviour for perturbed second order systems, preprint (1997).

[BB] M.L. Bertotti and S. Bolotin, A variational approach for homoclinics in almost periodic Hamiltonian systems, Comm. Appl. Nonlinear Analysis 2 (1995), 43-57.

[Bol] S. Bolotin, Existence of homoclinic motions, Vestnik Moskov. Univ. Ser. I Mat. MeKh. 6 (1980), 98-103.

[BS] B. Buffoni and E. Séré, A global condition for quasi random behaviour in a class of conservative systems, Comm. Pure Appl. Math. XLIX (1996), 285-305.

[CM] P. Caldiroli and P. Montecchiari, Homoclinics orbits for second order Hamiltonian systems with potential changing sign, Comm. Appl. Nonlinear Analysis 1 (1994), 97-129. 
[CS] K. Cieliebak and E. Séré, Pseudo-holomorphic curves and the shadowing Lemma, Duke Math. J. 77 (1995), 483-518.

[CZES] V. Coti Zelati, I. Ekeland and E. Séré, A variational approach to homoclinic orbits in Hamiltonian systems, Math. Ann. 288 (1990), 133-160.

[CZMN] V. Coti Zelati, P. Montecchiari and M. Nolasco, Multibump homoclinic solutions for a class of second order, almost periodic Hamiltonian systems, Nonlinear Diff. Eq. Appl. 4 (1997), 77-99.

[CZR] V. Coti Zelati and P.H. Rabinowitz, Homoclinic orbits for second order Hamiltonian systems possessing superquadratic potentials, J. Amer. Math. Soc. 4 (1991), 693-727.

[DPF] M. Del Pino and P.L. Felmer, Multi-peak bound states for nonlinear Schrödinger equations, Ann. Inst. H. Poincaré, Anal. Non Linéaire, to appear.

[G] C. Gui, Existence of multi-bump solutions for nonlinear Schrödinger equations via variational methods, Comm. in PDE 21 (1996), 787-820.

[HW] H. Hofer and K. Wysocki, First order elliptic systems and the existence of homoclinic orbits in Hamiltonian systems, Math. Ann. 288 (1990), 483503.

[L] Y.Y. Li, On a singularly perturbed elliptic equation, preprint (1996).

[M] V.K. Melnikov, On the stability of the center for periodic perturbations, Trans. Moskov Math. Soc. 12 (1963), 1-57.

[MS] K.R. Meyer and G.R. Sell, Melnikov trasforms, Bernoulli bundles, and almost periodic perturbations, Trans. Amer. Math. Soc. 314 (1989), 63105.

[MN] P. Montecchiari and M. Nolasco, Multibump solutions for perturbations of periodic second order systems, Nonlinear Anal. TMA 27 (1996), 13551372.

[MNT1] P. Montecchiari, M. Nolasco and S. Terracini, Multiplicity of homoclinics for time recurrent second order systems, Calculus of Variations, to appear.

[MNT2] P. Montecchiari, M. Nolasco and S. Terracini, A global condition for periodic Duffing-like equations, Trans. Amer. Math. Soc., to appear.

[PT] J. Palis and F. Takens, Hyperbolicity \& sensitive chaotic dynamics at homoclinic bifurcations, Cambridge Studies in Advanced Mathematics 35, (1993).

[P] K.J. Palmer, Exponential dycotomies and transversal homoclinic points, J. Diff. Eq. 55 (1984), 225-256. 
[R1] P.H. Rabinowitz, Homoclinic orbits for a class of Hamiltonian systems, Proc. Roy. Soc. Edinburgh 114 A (1990), 33-38.

[R2] P.H. Rabinowitz, Multibump solutions for an almost periodically forced singular Hamiltonian system, Electronic Journal of Differential Equations 1995, No. 12 (1995).

[R3] P.H. Rabinowitz, A multibump construction in a degenerate setting, preprint (1996).

[Sc] J. Scheurle, Chaotic solutions of systems with almost periodic forcing, ZAMP 37 (1986), 12-26.

[S1] E. Séré, Existence of infinitely many homoclinic orbits in Hamiltonian systems, Math. Zeit. 209 (1991), 27-42.

[S2] E. Séré, Looking for the Bernoulli shift, Ann. Inst. H. Poincaré, Anal. Non Linéaire 10 (1993), 561-590.

[STT] E. Serra, M. Tarallo and S. Terracini, On the existence of homoclinic solutions for almost periodic second order systems, Ann. Inst. H. Poincaré, Anal. Non Linéaire 13 (1996), 783-812.

[Sp] G. Spradlin, Multibump solutions to a Hamiltonian system with an almost periodic term, preprint (1995).

[T] K. Tanaka, Homoclinic orbits in a first order superquadratic Hamiltonian system: convergence of subharmonic orbits, J. Diff. Eq. 94 (1991), 315-339.

[W1] S. Wiggins, Global bifurcation and chaos, Applied Mathematical Sciences, Springer-Verlag, 73 (1988).

[W2] S. Wiggins, On the detection and dynamical consequences of orbits homoclinic to hyperbolic periodic orbits and normally hyperbolic invariant tori in a class of ordinary differential equations, SIAM J. App. Math. 48 (1988), 262-285.

[WH] S. Wiggins and P. Holmes, Homoclinic orbits in slowly varying oscillators, SIAM J. Math. Anal. 18 (1987), 612-629. 